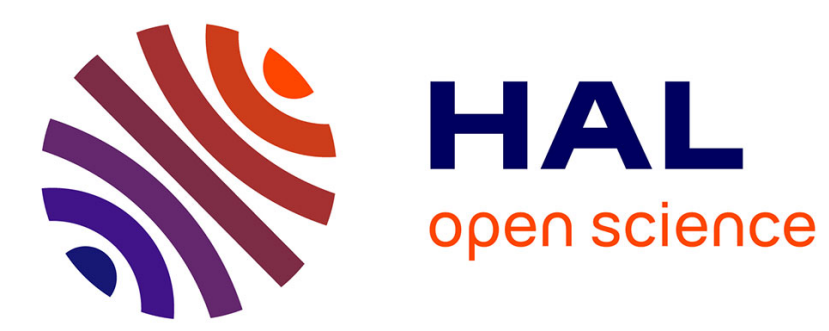

\title{
Simulation of the d.c. critical current in superconducting sintered ceramics
}

R. Riedinger, P. Habig, El-Kebir Hlil, M. Arnaud, C. Boulesteix

\section{To cite this version:}

R. Riedinger, P. Habig, El-Kebir Hlil, M. Arnaud, C. Boulesteix. Simulation of the d.c. critical current in superconducting sintered ceramics. Revue de Physique Appliquée, 1990, 25 (1), pp.67-72. 10.1051/rphysap:0199000250106700 . jpa-00246164

\section{HAL Id: jpa-00246164 https://hal.science/jpa-00246164}

Submitted on 1 Jan 1990

HAL is a multi-disciplinary open access archive for the deposit and dissemination of scientific research documents, whether they are published or not. The documents may come from teaching and research institutions in France or abroad, or from public or private research centers.
L'archive ouverte pluridisciplinaire HAL, est destinée au dépôt et à la diffusion de documents scientifiques de niveau recherche, publiés ou non, émanant des établissements d'enseignement et de recherche français ou étrangers, des laboratoires publics ou privés. 
Classification

Physics Abstracts

$74.60 \mathrm{~J}-64.60 \mathrm{C}-74.70 \mathrm{~V}-74.75$

\title{
Simulation of the d.c. critical current in superconducting sintered ceramics
}

\author{
R. Riedinger $\left({ }^{1}\right)$, P. Habig $\left({ }^{1}\right)$, E. K. Hlil $\left({ }^{1}\right)$, M. Arnaud $\left({ }^{2}\right)$ and C. Boulesteix $\left({ }^{2}\right)$ \\ (1) Laboratoire de Physique du Solide, Univ. de Haute Alsace, 4 rue des Frères Lumière, 68093 Mulhouse \\ Cedex, France \\ (2) Laboratoire de Microscopie Electronique Appliquée (CNRS UA 797), case B 61, 13397 Marseille Cedex, \\ France
}

(Reçu le 28 mai 1989, révisé le 5 septembre 1989, accepté le 12 septembre 1989)

\begin{abstract}
Résumé. - Les nouvelles céramiques frittées supraconductrices à haute température critique peuvent être décrites dans certains cas comme un réseau de tiges interconnectées, dans d'autres cas, comme un empilement plus ou moins aléatoire de cristallites parallélépipédiques ; leur taille est de l'ordre de quelques microns. Le courant critique continu à voltage nul d'un tel matériau n'est pas relié aux propriétés supraconductrices massives, mais à la structure granulaire du matériau. Le courant critique entre deux grains voisins est régi par le courant critique de leur jonction commune, qui se comporte comme une jonction Josephson, dans certaines limites. Dans ce travail, nous modélisons le système comme un réseau de jonctions Josephson. Nous spécialisons au cas du courant critique continu à voltage nul d'un réseau de jonctions identiques, immergé dans un espace à deux dimensions. Nous examinons l'influence des dimensions finies de l'échantillon. Nous discutons les relations avec la conductivité normale et la percolation.
\end{abstract}

\begin{abstract}
The new superconducting high- $T_{\mathrm{c}}$ sintered ceramics can be described in some case as a lattice of interconnected rods, in other cases as a more or less random packing of parallelepiped crystallites ; their size is about a few microns. The d.c. critical current at zero voltage of such a material is not related to the critical current of the bulk material, but to its granular structure. Indeed, the critical current between two adjacent cells is governed by the critical current of the weak link between them ; this link behaves within some limits as a Josephson junction, the critical current of which is known. For our present problem, the system can be modeled as a lattice of Josephson junctions. We present here results for the d.c. critical current at zero voltage of lattices of identical Josephson junctions in two dimensions. The influence of the finiteness of size of the sample is examined. The relationship with normal conductivity simulations and percolation is discussed.
\end{abstract}

\section{Introduction.}

The determination of the maximal critical d.c. current density at zero voltage in the new granular high$T_{\mathrm{c}}$ superconductive ceramics [1] is a problem of uppermost interest, as well for technological applications (the transportation without loss of electric power), as for theoretical ones (percolation above the threshold). According to their density and packing, the sintered superconductive materials are described either as a lattice of interconnected rods [2, SSG] in the case of low-density materials, either as a random packing of parallelepiped crystallites of typical size a few microns [3] in the case of denser ones.

In the first case (low density materials), a description within the Ginzburg-Landau theory is appropriate, if the superconducting correlation length $\xi_{\mathrm{s}}$ is larger than the lateral rod dimension [4]. Such an approach has been used by Leath and Tang [5] for simulating the maximal critical current, the voltage versus current behavior and the breakdown to normal state, on a bond percolation square lattice. 
For more compact materials we will be studying here, the critical d.c. current is limited by the weak links between grains [6] ; these are usually described as Josephson junctions. Again, such networks are a subject of intense interest, under magnetic field specially [7].

The critical current of a Josephson junction is given by :

$$
J_{\mathrm{c}}=\frac{1}{R} \frac{\pi}{2} \frac{\Delta(T)}{e} \tanh \frac{\Delta(T)}{2 k_{\mathrm{B}} T}
$$

$R$ is the resistance of the link in the normal state; $\Delta$ is the temperature dependent gap energy ; the last tanh factor describes the depairing of Cooper pairs caused by thermal excitation [8].

\section{The model.}

As described above, we model the sintered materials as a network of Josephson junctions. Each interface between two neighboring grains constitutes a junction (its critical current value is about $1 \mathrm{~mA}$ ). These Josephson junctions located at the boundary of a same grain are interconnected by this grain, which ought to be a bulk superconductor and to possess a higher critical d.c. current than all junctions at its boundary together.

In the following, according to the terminology of networks $[9,10]$, a Josephson junction will be denoted as a « node » or « site ", its critical current as its « node capacity »; the connection between two junctions located at the boundary of a same grain will be denoted as an « arc », and the « arc capacity » is not bounded here in a first approach.

For a lot of reasons, as well of physical as of chemical nature, one expects that the critical current of junctions is widely distributed between a maximal value, and a minimal one (usually zero). For instance, all junctions at the boundary of a nonsuperconducting grain (a foreign phase) will be defective, and possess a vanishing critical current ; chemical alteration of the interfaces (the oxygen surface concentration deviating from the bulk concentration optimal for superconductivity [11] will also strongly reduce the maximal critical current of the junctions located at these interfaces. Geometrical factors, like grain size and shape distribution, their relative orientation (texture) will also generate wide distributions for the critical currents of junctions, owing to the distribution of intergrain normal resistance [12] (cf. [1]).

Within a classical model neglecting the phase fluctuations of the superconducting order parameter between the grains, the maximal critical current of a composite and granular wire will be determined by the maximal superconducting flux crossing the associated network (as described above), compatible with the constraints that nowhere a capacity (critical current of a junction) should be exceeded. Otherwise, some of the junctions flip to the normal state and dissipate heat ; the behavior of the material in such a situation depends on the caloric coefficients of the material and embedding medium [5]. If we introduce the mild restriction that all capacities are integers, we are able to solve the problem of the determination of the maximal critical current (integer also) by the maximal flow algorithm of FordFulkerson [9] in the associated capacitated network. It is one of the outstanding results in econometrics and linear integer programming.

The Ford-Fulkerson theorem in its usual form is concerned with the maximal flow in networks with capacitated arcs and nodes with unrestricted capacity. We transform our problem into this canonical one by replacing a node with capacity $k$ by a pair of nodes with unrestricted capacity, linked by an arc of capacity $k[10]$ (Fig. 1).
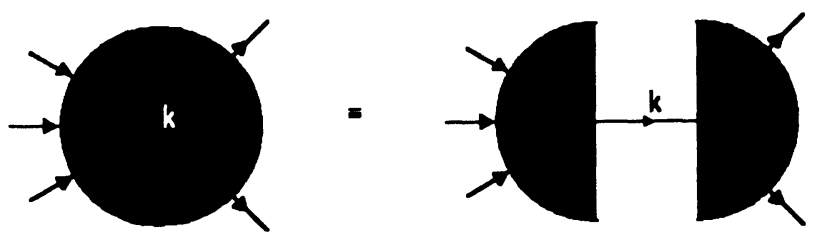

Fig. 1. - Transformation to canonical network for capacitated nodes. A node with capacity $k$ is replaced by two nodes of unrestricted capacity, connected by an arc of capacity $k$ grain (dotted).

The conclusions which may be drawn at this point are the following :

i) we may simplify the network by removing all dead sites, isolated islands and dead ends (primary ineffective junctions, sites not connected to any electrode or to one only), leaving over the backbone of sites and arcs connected to both electrodes (called source and sink in the network terminology);

ii) the maxflow-mincut theorem of Ford-Fulkerson states that the maximal flow corresponds to a minimal cut across the network (this is the sum of capacities of the arcs cut in order to split the network into two pieces) [9] ;

iii) this solution is numerical ; it is independent of the topology and dimensionality of the space.

We will examine first the critical current in the simplest networks of Josephson junctions, a « site percolation » system. We assume that a proportion $c$ of junctions (sites) is defective, with vanishing critical current $(0)$, and that all active junctions are identical with the same critical current (1).

Without restriction, we may assume that the capacity of all arcs interconnecting the sites (junctions) is unity also. This class of networks has unit 
arc capacity everywhere and is furthermore simple (i.e. each node has inflow or outflow bounded by one). Special results exist for such networks, namely accelerated convergence for Ford-Fulkerson implementations, which can be run in a time of order $\left(v^{1 / 2} a\right)$, where $v$ is the total number of sites, $a$ the total number of arcs [10].

There exists also a relation for the distance from source $s$ to sink s' (the electrodes), i.e. the minimal number of arcs to be crossed from one to the other :

$$
d\left(\mathrm{~s}, \mathrm{~s}^{\prime}\right) \leqslant v / I_{\mathrm{c}},
$$

where $v$ is the total number of nodes of the network, $I_{\mathrm{c}}$ the maximal flow, $d$ the distance (in steps) between the two electrodes [10].

\section{Two-dimensional networks.}

We present here results corresponding to the critical d.c. current for two-dimensional lattices, a band of width $b$ and length $n$ wrapped onto a cylinder (periodic boundary conditions on b) ; we simplify somewhat the connections, by keeping in a first stage only a subset of one-way arcs, in an oriented network. These assumptions are unessential for the method, but not for the results concerning the percolation, since the critical concentration and the critical exponents are dependent on the fact that the arcs are directed or not [13-15]; evenmore, the «susceptibility » exponents are anisotropic :

a) the triangular lattice : each site is connected to two sites on its right, as shown in figure 2 ;

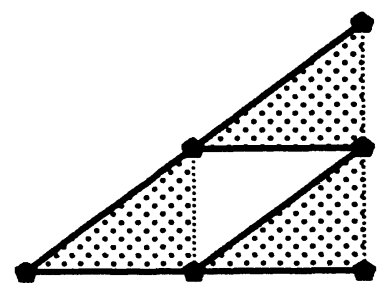

Fig. 2. - Triangular lattice. Each grain has a triangular shape ; each vertex is connected to two vertices at its right (bold arc) in each grain (dot-filled).

b) a lattice of «bricks »: it is a variant of a), which looks more like superconducting sintered materials (Fig. 3).

In figure 4 , we represent such a typical configuration, for a given concentration of defective sites. The most stringent feature is that even in the backbone, not all sites and arcs may be used simultaneously, due to the bottleneck created by the minimal cut somewhere in the lattice.

We recall that the maximal flow has a unique solution associated with a unique minimal cut (up to

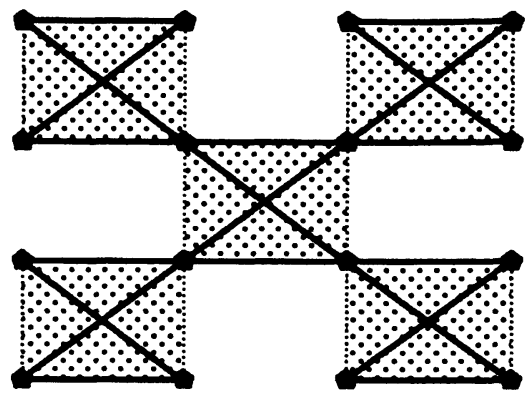

Fig. 3. - A lattice of "bricks». Each grain has a rectangular shape; each vertex is connected to two vertices at its right (bold line) in each grain (dot-filled). There is no connection between arcs not crossing at a vertex.

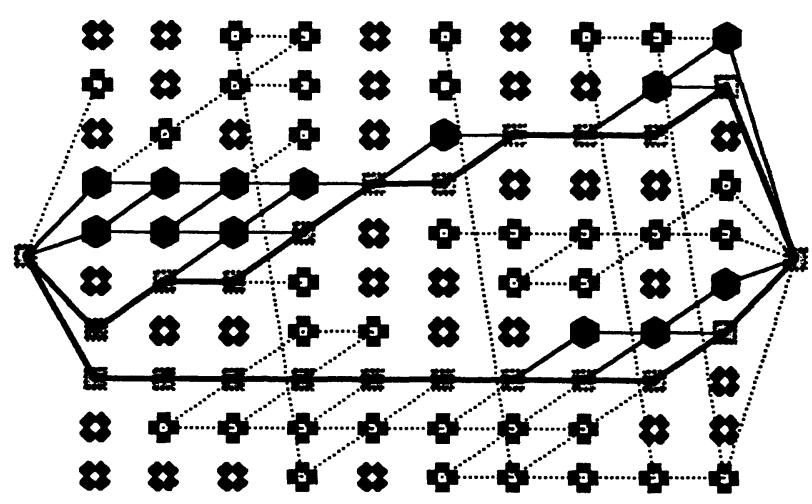

Fig. 4. - A flow in a triangular lattice. The size of the lattice is $10 \times 10$ (width $b$, depth $n$ ); the concentration of defects is 0.35 . Squares : connected active junctions; hexagons : connected inactive junctions $;(x)$ : primary killed junctions ; $(+)$ : partially or fully disconnected junctions ; bold line : a current path ; normal line : unused arcs ; dotted line : partially connected arcs. The two sites at the left and right represent the electrodes (source and sink resp.). Note the periodic boundary conditions on the links from the upper to the lower sides.

accidental degeneracy); the cut is a «surface » cutting the network into two pieces such that the sum of capacities of arcs crossing this "surface " is minimal. Many configurations of the flow lead to this optimal solution for the current. The physical ow wou e an a equa e average over a ese flows ; but this is irrelevant for our present problem, the determination of the maximal critical current.

In figure 5, we report the results for the critical current versus concentration of defective junctions, for various choices of $b$ and $n$; an average over one hundred configurations per concentration has been performed, and the computational fluctuation has been recorded.

For all perfect configurations, the maximal critical current $I_{\mathrm{c}}$ is $b$. This corresponds to the limit of the inequality (2), with $d\left(\mathrm{~s}, \mathrm{~s}^{\prime}\right)=n$, and $v=n b$. We 


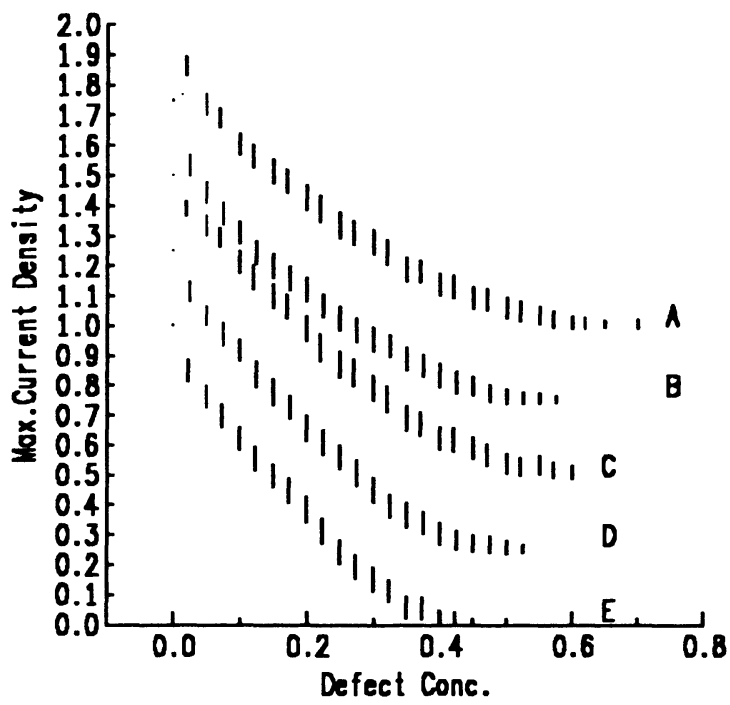

Fig. 5. - Critical current and variance for 2d-lattices. Critical current density $\left\langle i_{\mathrm{c}}\right\rangle=\frac{1}{N} \sum I_{\mathrm{c}} / b$ and variance $\left(\frac{1}{N} \sum\left(i_{\mathrm{c}}-\left\langle i_{\mathrm{c}}\right\rangle\right)^{2}\right)^{1 / 2}$, where $b$ denotes the width, $N$ the number of configurations. A : Bricks $10 \times 10$; B : Bricks $10 \times 20$; C: Triangles $10 \times 10 ; \mathrm{D}$ : Triangles $10 \times 20 ; \mathrm{E}$ : Triangles $10 \times 30$. The curves A, B, C, D, E are shifted upwards for a better readability of $1.0,0.75$, $0.50,0.25,0.0$ respectively. Note that the critical concentration diminishes with the ratio $b / n$, and the critical current density scales accordingly.

therefore reported the current density $i_{\mathrm{c}}=I_{\mathrm{c}} / b$ in 2d.

At very low concentration of defects, the maximal current is linear versus the concentration, since each defective node (junction) removes a path.

There exists a threshold concentration $c^{*}$ above which the current vanishes. This threshold coincides with the percolation limit (probability that there exists at least a path crossing the network [16]). Near the threshold, the path is unique and self-avoiding. For finite samples, the percolation limit depends on the size and shape of the sample, and is shifted from its limit value in an infinite system. Extrapolation procedures are exposed in references [14, 17]. According to the SSG model [2] adapted to superconductors [18], near the concentration threshold, the critical current should scale as a power law :

$$
i_{\mathrm{c}}= \begin{cases}\alpha\left(c^{*}-c\right)^{\beta}, & c \leqslant c^{*} \\ 0, & c>c^{*}\end{cases}
$$

We analyzed our data in that model; but rather than extracting the critical exponents of the percolation region near the cutoff (our actual data are too scarce for that purpose), we merely checked that such a law is accurate quite over the whole concentration range. The exponents obtained in such a fit are somewhat sensitive to the threshold concentration and the domain fitted. We recall that the aim of this work is to explore the domain above the percolation threshold; the results are reported in table I.

Table I. - Fit of critical current densities versus concentration to a power lax. We compare our results with results existing in the litterature for the percolation threshold. If we restrict our fit to the concentration domain near the percolation limit, our exponents become closer of those of the percolation limit.

\begin{tabular}{|l|l|c|c|c|}
\hline \multicolumn{1}{|c|}{ Model ( $\dagger$ ) } & \multicolumn{1}{c|}{$\mathrm{c}^{*}$} & $\alpha$ & $\beta$ & Fig. 4 \\
\hline $\mathrm{B}, 10 \times 10$ & 0.70 & 1.81 & 1.84 & $\mathrm{~A}$ \\
$\mathrm{~B}, 10 \times 20$ & 0.588 & 2.42 & 2.02 & $\mathrm{~B}$ \\
\hline $\mathrm{T}, 10 \times 10$ & 0.65 & 2.2 & 2.0 & $\mathrm{C}$ \\
$\mathrm{T}, 10 \times 20$ & 0.56 & 3.9 & 2.3 & $\mathrm{D}$ \\
T, 10 $\times 30$ & 0.47 & 1.3 & 1.9 & $\mathrm{E}$ \\
\hline Ref. [18] 2D & & & 1.25 & \\
Ref. [18] 3D & & & 1.75 & \\
Note 1 & 0.7035 & & 1.733 & \\
Note 2 & 0.5949 & & 1.733 & \\
\hline
\end{tabular}

(†) B refers to the lattice of bricks, $\mathrm{T}$ to the triangular lattice.

Note $1:$ directed 2-dim. square lattice, site percolation [13] ; $\beta$ refers to the parallel coefficient ; cf. also [14, 15].

Note 2: directed 2-dim. triangular lattice, site percolation [13].

\section{Conclusion.}

In this paper, we showed how granular sintered superconducting ceramics may be modeled as a network of Josephson junctions. Within the classical model neglecting the phase fluctuations of the superconducting order parameter defined in each grain, we showed that the Ford-Fulkerson theorem and the associated algorithms is a very efficient way to determine the critical superconducting current, independently of the dimension of space, with the sole limitation of integrity on capacities and flows. The main result, that the maximal flow (critical current) is limited by the minimal cut (an interface cutting the network into two pieces with minimal throughput) is immediate. The network model is independent of the real dimension of the space. Here, we applied just our model to the generalized site percolation on two-dimensional directed triangular- and square lattices. This is a more general problem than the usual percolation, since we have to find out the maximal number of correlated non-intersecting pathes crossing a network for a given concentration of defective 
junctions. This is related to directed self-avoiding walks in polymer physics [19].

It is possible to fit the critical current density to a power law (such a power law is strictly valid near the percolation threshold only [18]) over the whole concentration range; but the exponents obtained in this way are slightly higher than those obtained for a neighborhood of the threshold concentration only. All these parameters are size- and shape dependent, and accurate determinations would require bigger samples and more refined extrapolation procedures $[17,14]$.

If the percolation has been well studied on mixtures of classical superconductors and normal materials [18], only very few experimental results exist for new ceramics about the dependence of the critical current versus the concentration of defective junctions [20]. In order to correlate to properties like texturing [5] or interface oxygen stoichiometry in ceramics [11], one has to rely the maximal critical current of a junction to the physical and chemical parameters. We considered here intergrain junctions ; but the model may be useful for inner grain junctions also [21]. We hope to extend this work to directed and non directed networks in two and three dimensions, taking into account of other physical and chemical parameters, and randomness [22]. Extensions to quantum aspects like localization [23] or vortex flux lines remain for the moment out of scope.

\section{Acknowledgments.}

The computations have been performed in APL at CCSC (service commun CNRS) within the project «Calcul Numérique Intensif ». We whish to thank Dr S. Roux (ENSPCI, Paris) for fruitful discussions about directed percolation.

Note added in revised version. J. Rhyner and G. Blatter (Phys. Rev. B 40 (1989) 829) examined independently the problem of maximal critical current on textured materials along similar ideas, in the framework of linear programming and duality between the maximal flow and the minimal cut [10], without the integrity assumption about capacities and currents. However, the numerical convergence of the simplex method they are using is exponential, and is less effective than efficient implementations of the Ford-Fulkerson algorithm, which requires the integrity condition, but can be run in a time polynomial in the size of the system.

\section{References}

[1] High $T_{\mathrm{c}}$ Superconductors Materials and Mechanims of Superconduction, Eds J. Müller and J. L. Olsen (North-Holland) 1988, Vols. 1, 2.

[2] DE Gennes P. G., J. de Phys. Lett. France 37 (1976) L1;

Skal A. S. and Shrlovski B. J., Fiz. Tekh. Poluprodn. 8 (1974), 1856 (Sov. Phys. Semicond. 8 (1975) 1029).

[3] Farrell D. E., Chandrasekhar B. S., MC Guire M. R., FANG M. M., Kogan V. G., Clem J. R. and Finnemore D. K., Phys. Rev. B 36 (1987), 4025 ;

Ginley D. S., Venturini E. L., Kwack J. F., BAUghMAN R. J., MOROSIN B. and SCHIRBER J. E., Phys. Rev. B 36 (1987) 829.

[4] Fink H. J., Lopez A. and MAYNARd R., Phys. Rev. B 26 (1982) 5237 ;

ammal R., Lubensky T. C. and Toulouse G., Phys. Rev. B 27 (1983) 2820.

[5] Leath P. and Tang W., Phys. Rev. B 39 (1989) 6484.

[6] Likharev K. K., Rev. Mod. Phys. 51 (1979) 101 ; Senoussi S., Oussena M., Ribault M. and Collin G., Phys. Rev. B 36 (1987) 4003.

[7] Shin W. Y. and Stroud D., Phys. Rev. B 32 (1985) 158 ;

NiU Q. and Nori F., Phys. Rev. B 39 (1989) 2134.

[8] Ambegaokar V. and Baratoff A., Phys. Rev. Lett. 10 (1963) 486 ;

erratum: ibidem 11 (1963) 104.
[9] Ford L. R. and Fulkerson D. R., Flows in Networks (Princeton Univ. Press, Princeton) 1962 ;

GALE D., The Theory of Linear Economic Models (MacGraw-Hill, New York) 1960.

[10] Papadimitriou Ch. H. and Steiglitz K., Combinatorial Optimization, Algorithms and Complexity (Prentice Hall, Englewood Cliffs) 1982, chap. 9.

[11] Panson A. J., Braginski A. I., Gavaler J. R., Hulen J. K., Janocko M. A., Pole H. C., Stewart A. M., TAlVacchio J. and Wagner G. R., Phys. Rev. B 35 (1987) 8774 ; Phys. Rev. B 39 (1989) 6484 .

[12] Dimos D., Chaudari P., Mannhart J. and Le Goues F. K., Phys. Rev. Lett. 61 (1988) 219 ;

Mannhart J., Chaudari P., Dimos D., Tsuei C. C. and MC Guire T. R., Phys. Rev. Lett. 61 (1988) 2476.

[13] De Bell K. and Essam J. W., J. Phys. A 16 (1983) 385.

[14] Roux S., J. Phys. A 20 (1987) L873.

[15] KINZEL, in Percolation, structures and processes, Ann. Israel Phys. Soc. 5, Eds. G. Deutscher, R. Zallen and J. Alder (Adam Highler, Bristol) 1983.

[16] Shante V. K. S. and KirkPatrick S., Adv. Phys. 20 (1971) 325.

[17] KirkPatrick S., in Ill Condensed Matter, Eds. R. Balian, R. Maynard and G. Toulouse (les 
Houches 1978, session XXXI), North-Holland (1979) p. 320 ; J. Phys. Lett. France 40 (1979) L219;

[18] Deutscher G. and Rappaport M. L., J. Phys. Colloq. France 39 (1978) C6-581;

Deutscher G., Chance and Matter, Eds. J. Souletie, J. Vannimenus and R. Stora (les Houches 1986, session XLVI) North-Holland (1987) p. 1.

[19] Rammal R., Toulouse G. and Vannimenus J., $J$. Phys. France 45 (1984) 389 ;

Knezevic M. and Vannimenus J., Phys. Rev. B 35 (1987) 4988.
[20] Goldschmidt D., Phys. Rev. B 39 (1989) 9139 ; Ogale S. B., DiJkKamp D., Venkatesen T., Wu X. D. and InAm A., Phys. Rev. B 36 (1987) 7210.

[21] Pande C. S., Singh A. K., Toth L. Gubser D. U. and Wolf S., Phys. Rev. B 36 (1987) 5669.

[22] Deutscher G. and Müller K. A., Phys. Rev. Lett. 59 (1987) 1745.

[23] Doniach S., in Percolation, Localization and Superconductivity, Eds. A. M. Goldman and S. A. Wolf, Nato ASI Series B, vol. 109 (Plenum) 1983, p. 401. 